\title{
Internalisierung von externen Effekten im Umweltbereich als Mittel der Regionalpolitik
}

\section{Einleitung}

Im Zentrum des vorliegenden Beitrages stehen externe Effekte im Umweltbereich oder Umweltexternalitäten. Er ist im Grenzbereich zwischen Wirtschaftsgeographie/Regionalforschung und Umweltökonomie anzusiedeln. Externe Effekte sind ein wirtschaftswissenschaftliches Konzept. Allgemein werden Kosten, die vom Verursacher auf Drittpersonen abgewälzt werden, als negative externe Effekte bezeichnet. Positive externe Effekte liegen vor, wenn jemand Nutzen oder Erträge für Dritte erbringt, ohne dafür bezahlt zu werden. Eine Sonderform stellen die sog. Spillovers dar. Dabei handelt es sich um räumliche externe Effekte, d. h. um positive und negative Auswirkungen einer Region auf andere Regionen, für welche keine Abgeltung bezahlt wird. Positive Umweltexternalitäten sind Umweltgüter und -dienstleistungen, die von den Nutznießern gratis oder unter dem Wert bezogen werden. Dabei ist konkret etwa an die Schutzwirkungen des Waldes (Lawinen-, Wasserschutz usw.), die Erholungswirkung einer schönen Landschaft usw. zu denken. Negative Umweltexternalitäten sind Umweltbelastungen (z. B. Luftverschmutzung), deren Kosten (Schadens-, Vermeidungs-, Reparaturkosten) nicht von den Akteuren selbst getragen werden, sondern auf Dritte abgewälzt werden. Aus Sicht der Umweltökonomie stellen Umweltexternalitäten zunächst einmal eine wesentliche Ursache für die Umweltproblematik dar, weil bei Vorliegen externer Effekte der Umwelt bei Handlungsentscheiden ein zu geringes Gewicht zukommt; es besteht ein zu geringer Anreiz, die Umwelt zu schützen, bzw. ein zu großer Anreiz, umweltschädigende Handlungen auszuführen. Hier wird jedoch die regionalwirtschaftliche Bedeutung der Umweltexternalitäten thematisiert. Es werden einerseits die regionalwirtschaftlichen Auswirkungen der Umweltexternalitäten analysiert, andererseits wird auf der Basis dieser Wirkungsanalyse eine Strategie «internalisierungsorientierter Regionalpolitik» entworfen, deren Grundidee vereinfacht dargestellt darin besteht, durch die Internalisierung externer Effekte, d. h. durch die Abgeltung positiver externer Effekte des ländlichen Raumes und durch die Anlastung negativer externer Effekte des städtischen Raumes, sowohl Anreize für Umweltschutz zu ver- mitteln als auch einen regionalen Ausgleich zu bewirken.

\section{Zur Bedeutung der externen Effekte in der Regionalforschung}

Nach BRUGGER/FISCHER (1985, 34 f.) stellen aus Sicht der Synthesearbeiten des Nationalen Forschungsprogrammes «Regionalprobleme in der Schweiz» die externen Effekte einen von vier wichtigen Ursachen- oder Schlüsselbereichen dar, die für die regionale Entwicklung relevant sind. Bei diesen Ursachen- oder Schlüsselbereichen handelt es sich erstens um regionale Potentiale und Ressourcen (z. B. Arbeitskräftepotentiale, Unternehmens-, Branchenstruktur, haushaltorientierte und unternehmensbezogene Infrastruktur), zweitens interregionale Austauschverhältnisse (z. B. Produktionsfaktorwanderungen, nachfrageseitige interregionale Kaufkraftströme, Ströme von Gütern, Dienstleistungen und Informationen), drittens Werte und Präferenzen der Akteure und viertens eben externe Effekte. Die Art und Weise, wie diese Bereiche im einzelnen und in ihren Beziehungen zueinander ausgestaltet sind, bestimmt im wesentlichen die Entwicklung einer Region.

In der regionalwissenschaftlichen Forschung wurden in der Vergangenheit vor allem wirtschaftsbezogene externe Effekte im Zusammenhang mit der wirtschaftlichen Entwicklungsproblematik thematisiert. So werden z. B. Agglomerationsprozesse teilweise mit positiven externen Effekten erklärt, die bei der Zusammenballung von Wirtschaft und Bevölkerung entstehen. Auf HOOVER (1948) geht die Untergliederung der externen Agglomerationsersparnisse in localization und urbanization economies zurück. Localization economies sind externe Ersparnisse, die durch das Vorhandensein mehrerer Betriebe derselben Branche entstehen (z. B. infolge vergrößerter Facharbeiterschaft, der Versorgung durch spezialisierte Zuliefer- und Reparatur-

Daniel Wachter, Dr., The World Bank, ENVPR S-3049, $1818 \mathrm{H}$ Street, N. W., Washington, D.C. 20433, USA 
betriebe usw.). Urbanization economies sind dagegen durch eine generell bessere Ausstattung mit städtischen Infrastrukturen und durch eine intensivere Verflechtung der wirtschaftlichen Aktivitäten bedingt. Ferner kommt in polarisationstheoretisch geprägten Wachstums- und Entwicklungstheorien Spillovers zwischen Zentrums- und Peripherieregionen eine große Bedeutung zu (vgl. z. B. MYRDALs [1974, 38 f.] Konter- und Ausbreitungseffekte oder PERROUXs [1964] Anstoß- und Bremseffekte).

Auf die regionalwirtschaftliche Bedeutung von $\mathrm{Um}$ weltexternalitäten wurde zwar bereits in verschiedenen Publikationen hingewiesen. Insgesamt ist aber aus dem Studium der regionalwissenschaftlichen und umweltökonomischen Literatur die Schlußfolgerung zu ziehen, daß die regionalwirtschaftlichen Auswirkungen der Umweltexternalitäten selten umfassend, d. h. unter Einbezug positiver und negativer Umweltexternalitäten, thematisiert wurden. Meist werden nur Teilaspekte diskutiert. Die ökonomische Literatur zur Thematik tendiert dazu, sich auf die negative Umweltexternalität (Luft-)Schadstoffemissionen zu beschränken (z. B. BENKERT 1981, SIEBERT 1978, 123 ff., SIEBERT u. a. 1980). Die regionalwirtschaftlichen Auswirkungen positiver Umweltexternalitäten kommen selten explizit zur Sprache. Die Literatur hierzu beschränkt sich meist auf die umweltbezogenen Wirkungen, wobei allerdings teilweise auch implizite Aussagen zu den regionalwirtschaftlichen Auswirkungen entnommen werden können (vgl. KRUTILLA/FISHER 1985, TSCHURTSCHENTHALER 1986).

\section{Zur regionalpolitischen Bedeutung von Umweltexternalitäten}

Anstelle einer allgemeinen Abhandlung (siehe dazu WACHTER 1990, 57-90) ziehen wir es vor, die regionalpolitische Bedeutung von Umweltexternalitäten im folgenden anhand von drei in der Literatur recht häufig erwähnten Sachverhalten darzulegen.

\section{Externe Agglomerationskosten}

In der ökonomischen Theorie der optimalen Stadtgröße (vgl. dazu RICHARDSON 1978, 73 f.) kommt den externen Agglomerationseffekten eine zentrale Bedeutung zu. Darin wird die Agglomerationsoder Stadtgröße mit dem Verhältnis von Kosten und Nutzen aus der Zusammenballung von Wirtschaft und Bevölkerung erklärt. Weiter oben wurde auf die externen Agglomerationserträge hingewiesen, die die Konzentration von Bevölkerung und Wirtschaft in Ballungsräumen fördern. Daneben fördern aber auch externe Agglomerationskosten Konzentrationsprozesse. Die Existenz von negativen Umweltexternalitäten (z. B. Luftverschmutzung und Verkehrsstaus) bedeutet, daß die Wirtschaftssubjekte nicht die vollen Kosten ihrer Handlungen tragen. Die Agglomerationsnachteile werden zu gering ausgewiesen, wodurch Ballungsräume über die volkswirtschaftlich optimale Größe hinauswachsen (vgl. dazu auch SIEBERT 1978, 138).

\section{Schadstoffspillovers}

BENKERT $(1981,113)$ beschreibt die hier vorliegende Problematik wie folgt: «Im Vergleich zweier ökologisch interdependenter Raumpunkte bedeutet das Wirken der Diffusionsfähigkeit allgemein, dass <externe> Assimilationskapazität mitgenutzt wird, ohne daß der Verursacher dieser Belastungen die ökologischen Kosten zu tragen hat. Für den betroffenen Raumpunkt verringert sich zugleich das verfügbare ökologische Potential. Die Erhöhung des Emissionsniveaus in einer Region bewirkt als ökonomische und ökologische Interdependenzen mit anderen Regionen, die die relative Wohlfahrtsposition der beteiligten Regionen dadurch in spezifischer Weise verändern, daß die auftretenden ökologischen Kosten intersektoral wie interregional von den Verursachern des erhöhten Emissionsniveaus abgewälzt werden können.» Ein klassisches Beispiel zu dieser Thematik ist die Rheinverschmutzung durch die verschiedenen Anrainerstaaten, welche in den Niederlanden hohe Wasseraufbereitungskosten verursacht.

\section{Positive externe Effekte des ländlichen Raumes}

Neben den negativen Umweltexternalitäten sind auch positive Umweltexternalitäten, welche überwiegend im ländlichen Raum zu lokalisieren sind, von regionalwirtschaftlicher Bedeutung. Bei den positiven externen Effekten handelt es sich wie erwähnt um Umweltgüter, wie z. B. die Schutzfunktionen des Waldes, die Erholungs- oder die ökologische Ausgleichsfunktion der Landschaft. Mit STORBECK $(1982,229-230)$ kann die Problematik wie folgt beschrieben werden: Die Funktionen des ländlichen Raumes erweisen sich bei näherer Prüfung «jedoch überwiegend als notwendige Ergänzungsfunktionen der Verdichtungsgebiete, die ohne solche Komplemente auch nicht existieren können . . . Der Vorrang, der (diesen Funktionen) eingeräumt wird, dient nicht der Stärkung der Entwicklungschancen dieser Regionen. Darum erfordert ein derartiges Konzept der räumlichen Funktionsteilung, daß der ländliche Raum für die Übernahme solcher Ergänzungsfunktionen auch angemessen honoriert werden muß.» Aus regionalwirtschaftlichen Gesichtspunkten sind also insbesondere wegen gesamträumlicher ökologischer Erfordernisse notwendige Nutzungsverzichte relevant. Teilweise sind allerdings die positiven externen Effekte auch mit direkten 
Kosten verbunden (die Sicherstellung der Schutzfunktionen des Waldes erfordert beispielsweise eine Waldpflege).

\section{Internalisierungsorientierte Regionalpolitik}

Oben wurde gezeigt, daß externe Effekte im Umweltbereich neben den umweltbezogenen Wirkungen auch regionalwirtschaftlich-raumstrukturelle Verzerrungen verursachen können. Es drängt sich daher der Gedanke auf, zu untersuchen, ob nicht durch die Internalisierung von externen Effekten, d. h. durch die Abgeltung von positiven und die Anlastung von negativen Umweltexternalitäten, neben verringerter Umweltbelastung auch ein regionaler Ausgleich bewirkt werden könnte. Diese Strategie kann als «internalisierungsorientierte Regionalpolitik» bezeichnet werden. Den Begriff prägte FREY $(1979,131)$, der allerdings nicht primär externe Effekte im Umweltbereich im Auge hatte, sondern externalisierte Ballungskosten im infrastrukturellen Bereich (Abwälzung von Kosten der Infrastrukturbereitstellung durch die zentralen Regionen auf die übrigen Regionen).

Bei der Begründung einer umweltbezogenen internalisierungsorientierten Regionalpolitik stellt sich allerdings das Problem, daß positive und negative Umweltexternalitäten häufig eng miteinander verknüpft sind. Wenn beispielsweise ein Landwirt eine ökologisch wertvolle, aber wenig ertragreiche Magerwiese intensiv zu nutzen beginnt, um auf ein gutes Einkommen zu kommen, verursacht er dadurch einerseits negative externe Effekte. Die Handlung kann aber auch als Folge davon interpretiert werden, daß er den Nutzen, den eine Magerwiese für die Gesellschaft stiftet, nicht abschöpfen kann (fehlende Internalisierung eines positiven externen Effektes).

Es wird somit die grundsätzliche Frage aufgeworfen, ob die Verursacher von Umweltbelastungen oder die Nutznießer intakter Umwelt für Umweltschutzkosten aufkommen sollen (Verursacherprinzip) vs. Nutznießer- oder Äquivalenzprinzip). Aus ökonomischer Sicht ist diese Frage im Prinzip offen. Denn ein wesentlicher Grund für die heutigen Umweltprobleme liegt in der Knappheit des Gutes Umwelt. Knappheit bedeutet, daß verschiedene, sich konkurrierende Verwendungsmöglichkeiten für das Gut Umwelt vorhanden sind. Entscheidend ist dabei, daß sämtliche an der Nutzungskonkurrenz $\mathrm{Be}$ teiligten für die Knappheit der Umwelt verantwortlich sind - sowohl Umweltbelaster als auch NutznieBer einer intakten Umwelt. Aus ökonomischer Sicht kann nicht a priori entschieden werden, welche Partei als Verursacherin der Umweltkosten zu betrachten ist und für die Umweltschutzkosten aufzukommen hat (COASE 1960).
Um in dieser Frage zu entscheiden, sind verschiedene Argumente in Betracht zu ziehen, z. B. ethische (in vielen Fällen würde es $\mathrm{z}$. B. als stoßend empfunden, wenn die Betroffenen die Urheber einer Umweltbelastung entschädigen müßten), verteilungspolitische (sind z. B. mit dem Verursacherprinzip unakzeptable soziale oder regionale Verteilungswirkungen verbunden?), juristische (bestehen traditionelle Eigentumsrechte, die unbedingt auch gegen umweltpolitisch motivierte Eingriffe zu schützen sind?) oder die Zweckmäßigkeit der Anreizwirkungen (eine Entschädigung für die Unterlassung einer Umweltbelastung kann problematisch sein, wenn dadurch eher ein Interesse an Subventionen als ein immanenter Anreiz für umweltfreundliches Handeln vermittelt wird).

Das Verursacherprinzip gilt zu Recht - neben dem Vorsorgeprinzip - als oberstes Leitprinzip im Umweltschutz, dafür sprechen insbesondere ethische Argumente und die ausgelösten Anreizwirkungen für umweltschonendes Handeln. Mit juristischen Argumenten (Einschränkung des Grundeigentums) kann allerdings die These vertreten werden, da $\beta$ bei boden- und landschaftsbezogenen Umweltproblemen (Natur- und Landschaftsschutz) das Äquivalenzprinzip angewendet werden sollte.

Es gibt in der schweizerischen Politik bereits einige diesbezügliche Ansätze. Es sei z. B. das Bundesgesetz über den Natur- und Heimatschutz erwähnt, das bei der Änderung vom 19. Juni 1987 mit Artikel $18 \mathrm{c}$ eine Grundlage für Abgeltungen an Grundeigentümer oder Bewirtschafter für Biotopschutz erhielt, oder die Verordnung über Bewirtschaftungsbeiträge an die Landwirtschaft unter erschwerten Produktionsbedingungen, die der Bundesrat am 20. Dezember 1989 abänderte. Die neuen Artikel 22-29 ermöglichen nun Bewirtschaftungsbeiträge für die Pflege von Trockenstandorten und Streueflächen durch Landwirte auch außerhalb von Erschwerniszonen. Solche relativ neuen Rechtsbestimmungen gilt es konsequent zu nutzen; auch andere Natur-/Landschaftsschutzbereiche müßten auf diese Weise geregelt werden können (z. B. Schutz schöner Landschaften vor Wasserkraftnutzung mittels des «Landschaftsrappens», also des Vorschlages der Schweizerischen Greina-Stiftung, Gemeinden und Kantone, die freiwillig auf eine Wasserkraftnutzung verzichten, dafür aus einem Fonds zu entschädigen, der mit einer Abgabe auf in der Schweiz produzierter Hydroelektrizität gespiesen würde).

Neben der Stoßrichtung «Äquivalenzprinzip» basiert die hier vorgeschlagene umweltbezogene internalisierungsorientierte Regionalpolitik (vgl. dazu auch WACHTER 1990) ferner auf einer zweiten Stoßrichtung, einer regionalisierten Umweltpolitik nach dem Verursacherprinzip. Im Bereich der übrigen Umweltschutzaufgaben, die nach dem Verursacherprinzip ausgestaltet sein sollten, empfiehlt sich aus Effi- 
zienzgründen eine regionale Differenzierung der Umweltpolitik, insbesondere eine restriktivere Umweltpolitik in Ballungsräumen, weil gezeigt werden kann, daß die externen Kosten in Verdichtungsräumen besonders hoch sind. Diese Stoßrichtung vermittelt mobilen Produktionsfaktoren Wanderungsanreize weg von den Großagglomerationen und setzt dadurch Dezentralisationspotentiale frei. Auch hierzu gibt es bereits einige Ansätze in der Schweiz, z. B. die Maßnahmenpläne gegen übermäßige Luftbelastungen gemäß Luftreinhalteverordnung vom 16. Dezember 1985 (Artikel 31-34), die die Kantone für Gebiete zu erarbeiten haben, in denen trotz der das ganze Land betreffenden allgemeinen Luftreinhaltemaßnahmen (z. B. Emissionsvorschriften für Autos) immer noch übermäßige Luftbelastungen auftreten. Diese Maßnahmenpläne müssen vor allem für Ballungsräume entworfen werden.

Mit den genannten beiden Stoßrichtungen können regionalpolitische Erfordernisse in der Umweltpolitik berücksichtigt werden, ohne daß damit ökologische Zielverluste in Kauf genommen werden müssen. Beide Stoßrichtungen lassen sich plausibel begründen und sind mit dem Kriterium der ökonomischen Effizienz vereinbar. Eine Vernetzung von Umwelt- und Regionalpolitik ist eine Notwendigkeit, da ohne die Berücksichtigung regionalpolitischer Anliegen in der Umweltpolitik viele Umweltschutzaufgaben mit regionalpolitischen Argumenten abgewehrt werden können. Andererseits wäre den ländlichen Regionen zu empfehlen, Umwelt-, Natur- und Landschaftsschutz vermehrt auch als Chance zu betrachten (insbesondere Abgeltungen für Nutzungsverzichte), und diese im Sinne einer Vorwärtsstrategie aktiv zu nutzen.

\section{Literatur}

BENKERT, W. (1981): Die raumwirtschaftliche Dimension der Umweltnutzung, Berlin.

BRUGGER, E. A., FISCHER, G. (1985): Regionalprobleme in der Schweiz: Grundzüge und Zusammenhänge. In: Fischer, G., Brugger, E. A. (Hrsg.) 1985: Regionalprobleme in der Schweiz - Ergebnisse eines Nationalen Forschungsprogrammes, Bern.

COASE, R. H. (1960): The nature of social cost. In: Journal of Law and Economics, Vol. 3, Chicago.

FREY, R. L. (1979): Die Infrastruktur als Mittel der Regionalpolitik, Bern.

HOOVER, E. M. (1948): The location of economic activity, New York u. a.

KRUTILLA, J. V., FISHER, A. C. (1985): The economics of natural environments, Washington D.C. (2. Auflage)

MYRDAL, G. (1974): Ökonomische Theorie und unterentwikkelte Regionen, Frankfurt am Main.

PERROUX, F. (1964): L'économie du XXe siècle, Paris (2. Auflage).

RICHARDSON, H. W. (1978): Urban economics, Hinsdale-III. SIEBERT, H. (1978): Ökonomische Theorie der Umwelt, Tübingen.

SIEBERT, H., WALTER, I., ZIMMERMANN, K. (Hrsg.) (1980): Regional environmental policy, New York.

STORBECK, D. (1982): Konzepte der Raumordnung in der Bundesrepublik. In: Akademie für Raumforschung und Landesplanung (Hrsg.), Grundriß der Raumordnung, Hannover.

TSCHURTSCHENTHALER, P. (1986): Das Landschaftsproblem im Fremdenverkehr - dargestellt anhand der Situation des Alpenraumes, Bern, Stuttgart.

WACHTER, D. (1990): Externe Effekte, Umweltschutz und regionale Disparitäten - Begründung und Ausgestaltungsmöglichkeiten einer internalisierungsorientierten Regionalpolitik. Reihe Wirtschaftsgeographie und Raumplanung, Nr. 9, Zürich. 